\title{
Human immunodeficiency virus evolution towards reduced replicative fitness in vivo and the development of AIDS
}

\author{
Dominik Wodarz $^{1, *}$ and David N. Levy ${ }^{2}$ \\ ${ }^{1}$ Department of Ecology and Evolutionary Biology, University of California, 321 Steinhaus Hall, Irvine, CA 92697, USA \\ ${ }^{2}$ Department of Basic Sciences, New York University College of Dentistry, 847 Schwartz Building, \\ 345 East 24th Street, New York, NY 10010, USA
}

\begin{abstract}
Human immunodeficiency virus (HIV) infection progresses to AIDS following an asymptomatic period during which the virus is thought to evolve towards increased fitness and pathogenicity. We show mathematically that progression to the strongest HIV-induced pathology requires evolution of the virus towards reduced replicative fitness in vivo. This counter-intuitive outcome can happen if multiple viruses co-infect the same cell frequently, which has been shown to occur in recent experiments. According to our model, in the absence of frequent co-infection, the less fit AIDS-inducing strains might never emerge. The frequency of co-infection can correlate with virus load, which in turn is determined by immune responses. Thus, at the beginning of infection when immunity is strong and virus load is low, co-infection is rare and pathogenic virus variants with reduced replicative fitness go extinct. At later stages of infection when immunity is less efficient and virus load is higher, co-infection occurs more frequently and pathogenic virus variants with reduced replicative fitness can emerge, resulting in T-cell depletion. In support of these notions, recent data indicate that pathogenic simian immunodeficiency virus (SIV) strains occurring late in the infection are less fit in specific in vitro experiments than those isolated at earlier stages. If co-infection is blocked, the model predicts the absence of any disease even if virus loads are high. We hypothesize that non-pathogenic SIV infection within its natural hosts, which is characterized by the absence of disease even in the presence of high virus loads, could be explained by a reduced occurrence of co-infection in this system.
\end{abstract}

Keywords: HIV dynamics; mathematical models; evolution; disease progression; coinfection; fitness

\section{INTRODUCTION}

AIDS develops when the CD4 T-cell count drops from normal levels ( 1000 cells $\mu 1^{-1}$ blood) to an average of 200 cells $\mu 1^{-1}$ blood. This occurs after an asymptomatic phase of varying length, most often after several years. Human immunodeficiency virus (HIV) evolution in vivo is thought to contribute to viral persistence and ultimately to disease progression. Experimental data from monkeys infected with simian immunodeficiency virus (SIV) clearly indicate that during disease progression new viral strains emerge that have the potential to induce increased amounts of pathology (Hirsch 1999; Kimata et al. 1999). It has been suggested that emergence of immune escape mutants contributes to an increase in viral load and disease progression in HIV and experimental SIV infection (Nowak et al. 1991; Phillips et al. 1991; Goulder et al. 1997; McMichael \& Phillips 1997; Price et al. 1997; Kimata et al. 1999). Generally, the onset of AIDS is thought to correlate with the emergence of virus strains characterized by increased replicative fitness (Kimata et al. 1999; Nowak \& May 2000).

Although virus evolution seems to induce progression to AIDS in HIV and experimental SIV infection, the

\footnotetext{
* Author for correspondence (dwodarz@uci.edu).
}

Electronic supplementary material is available at http://dx.doi.org/10. 1098/rspb.2007.0413 or via http://www.journals.royalsoc.ac.uk. situation seems different in monkeys infected naturally with SIV. In contrast to experimental SIV infection, monkeys that are naturally infected with SIV have had a history of coevolution with the virus. These animals can show viral profiles that are typical of AIDS in humans (i.e. high virus loads, high degrees of viral diversity, weak immunity), and yet remain healthy for life (Goldstein et al. 2000; Broussard et al. 2001; Silvestri et al. 2003).

Most theoretical studies about the in vivo evolution of $\mathrm{HIV}$ and disease progression have been performed with mathematical models which assume that a cell can be infected by only one virus particle. The reason for this was the assumption that cells downregulate the CD4 receptor shortly after becoming infected, preventing further viruses from entering the cell. Experimental data (Jung et al. 2002; Dang et al. 2004; Levy et al. 2004; Chen et al. 2005), however, argue that a cell can become infected with multiple virus particles, a process we call co-infection. While the CD4 receptor is eventually downregulated in infected cells, this takes a couple of days or so, which is a large enough time window for multiple viruses to infect the cell. Epidemiological work suggests that competition between pathogens, and thus the expected evolutionary trajectories, can be different if multiple pathogens infect the same host (Levin \& Pimentel 1981; Levin 1983a,b; Nowak \& May 1994). Similarly, in vivo virus competition and evolution can be expected to be changed in the context of co-infection. 
Here, we examine the effect of co-infection on viral evolution in vivo, and present a theory that might explain how viral evolution can lead to two alternative outcomes: (i) high virus load with the development of AIDS and (ii) high virus load without the development of AIDS. We suggest that, contrary to previous thinking, the development of AIDS requires the emergence of virus strains with reduced replicative fitness, and that this can be observed only if a sufficient degree of co-infection occurs, i.e. if a sufficient number of cells are infected by more than one virus. If co-infection is less frequent, however, disease-inducing virus strains fail to emerge, and we can observe absence of AIDS despite high virus loads.

This paper is written such that all the mathematical details are discussed in $\$ 2$, while the main text reports the results in a style that is also accessible to a biological readership.

\section{MATERIAL AND METHODS}

Here, the mathematical models and their basic properties are discussed. For an extended discussion of simplifying assumption and robustness, see electronic supplementary material. Because the values of the model parameters are largely unknown, the model was analysed in general terms, and parameter values in figures were chosen arbitrarily for illustrative purposes.

\section{(a) Basic model}

We start from a simple model of virus dynamics that is well established in the literature (Nowak \& May 2000). It contains the following variables: uninfected cells, $x$, and infected cells, $y$. Assuming that the population of free viruses turns over with a relatively fast rate and is in a quasi-steady state, the model is given by the following pair of differential equations:

$$
\begin{aligned}
& \frac{\mathrm{d} x}{\mathrm{~d} t}=\lambda-d x-\beta x y \quad \text { and } \\
& \frac{\mathrm{d} y}{\mathrm{~d} t}=\beta x y-(a+d) y
\end{aligned}
$$

Uninfected cells are produced with a rate $\lambda$, die with a rate $\mathrm{d} x$, and become infected with a rate $\beta x y$. Infected cells die with a rate ay. Because we assume that the virus population is in a quasi-steady state, the parameter $\beta$ summarizes the overall rate of viral replication, including the rate of virus production, the rate of infection and the death rate of free viruses. We further assume that an increase in the viral cytopathicity correlates asymptotically with a higher rate of virus production, and thus with a higher value of $\beta$. Hence, we replace the parameter $\beta$ by $\beta^{\prime}=f a /(g+a)$. The basic reproductive ratio of the virus is given by $R_{0}=\lambda \beta^{\prime} / \mathrm{d}(d a)$. If $R_{0}>1$, an infection is successfully established in the host, and the system converges to the following equilibrium:

$x^{*}=\frac{(a+d)}{\beta^{\prime}}, \quad y^{*}=\frac{\lambda}{(a+d)}-\frac{d}{\beta^{\prime}}$.

The total number of target cells at equilibrium, $x^{*}+y^{*}$, is a measure of the degree of pathology caused by the virus.

\section{(b) Co-infection model}

The co-infection model is given by the following set of differential equations:

$$
\begin{gathered}
\frac{\mathrm{d} x}{\mathrm{~d} t}=\lambda-\mathrm{d} x-\beta_{1}^{\prime} x\left(y_{1}+y_{12}\right)-\beta_{2}^{\prime} x\left(y_{2}+y_{12}\right), \\
\frac{\mathrm{d} y_{1}}{\mathrm{~d} t}=\beta_{1}^{\prime} x\left(y_{1}+y_{12}\right)-\left(a_{1}+d\right) y_{1}-\beta_{2}^{\prime} y_{1}\left(y_{2}+y_{12}\right)-p y_{1} z, \\
\frac{\mathrm{d} y_{2}}{\mathrm{~d} t}=\beta_{2}^{\prime} x\left(y_{2}+y_{12}\right)-\left(a_{2}+d\right) y_{2}-\beta_{1}^{\prime} y_{2}\left(y_{1}+y_{2}\right)-p y_{2} z, \\
\frac{\mathrm{d} y_{12}}{\mathrm{~d} t}=\beta_{1}^{\prime} y_{2}\left(y_{1}+y_{12}\right)+\beta_{2}^{\prime} y_{1}\left(y_{2}+y_{12}\right)-\left(a_{2}+d\right) y_{12}-p y_{12} z
\end{gathered}
$$

and

$$
\frac{\mathrm{d} z}{\mathrm{~d} t}=F\left(z, y_{1}+y_{2}+y_{12}\right)-b z
$$

The variables $y_{1}$ and $y_{2}$ denote the populations of cells infected only by virus 1 and virus 2 , respectively. The variable $y_{12}$ denotes cells that are co-infected by both viruses. The variable $z$ denotes a specific immune response that expands upon antigenic stimulation (such as B cell and $\mathrm{T}$ cells). We assume that virus 2 is more cytopathic than virus 1 (i.e. $a_{2}>a_{1}$ ). The model is an extension of previous work (Levin \& Pimentel 1981; Levin 1983a,b), with the addition of an immune response. Virus 1 replicates with a rate $\beta_{1}^{\prime}$, and virus 2 replicates with a rate $\beta_{2}^{\prime}$. As in the simple model (2.1), we assume that the viral cytopathicity and replication rate are related according to $\beta_{1}^{\prime}=f a_{1} /\left(g+a_{1}\right)$ and $\beta_{2}^{\prime}=f a_{2} /\left(g+a_{2}\right)$. Virus 1 is produced from cells $y_{1}$ and $y_{12}$, and virus 2 is produced from cells $y_{2}$ and $y_{12}$. Cells infected with virus 1 die with a rate $\left(a_{1}+d\right)$ and cells infected with virus 2 die with a rate $\left(a_{2}+d\right)$. Cells infected with both viruses show dominance and die with a rate $\left(a_{2}+d\right)$ because virus 2 is assumed to be more cytopathic than virus 1 . Immune responses expand upon exposure to all types of infected cells. One of the simplest ways to describe this is given by $F=c\left(y_{1}+y_{2}+y_{12}\right)$, where $c$ is the immune responsiveness (Nowak \& May 2000). Further, immune cells die with a rate $b$. While much more detailed models have been developed (De Boer \& Perelson 1998; Antia et al. 2005), our arguments hold for all models where a stronger immune response correlates with lower virus load. In the simplest form, the model assumes that immune responses kill infected cells with a rate $p$. In addition, simulations took into account non-lytic immune responses that inhibit viral spread by dividing the parameter $\beta^{\prime}$ by $z+\varepsilon$.

\section{RESULTS AND DISCUSSION}

\section{(a) Replicative fitness and the development of $A I D S$}

By replicative fitness, we mean the fitness of the virus based on viral parameters alone. It does not take into account the effect of anti-viral immunity. Therefore, as a starting point to developing a model of the relationships among replicative fitness, viral evolution and disease progression, we assume virus replication in the absence of immunity. The effect of immunity will be explored subsequently.

The well-established mathematical model of infection dynamics (Nowak \& May 2000; Perelson 2002), shown in $\S 2$, predicts that maximal reduction in the total number of host cells is brought about by a fast viral replication rate and an intermediate level of viral cytopathicity (rate of virus-induced cell killing; Wodarz \& Krakauer 2000). 


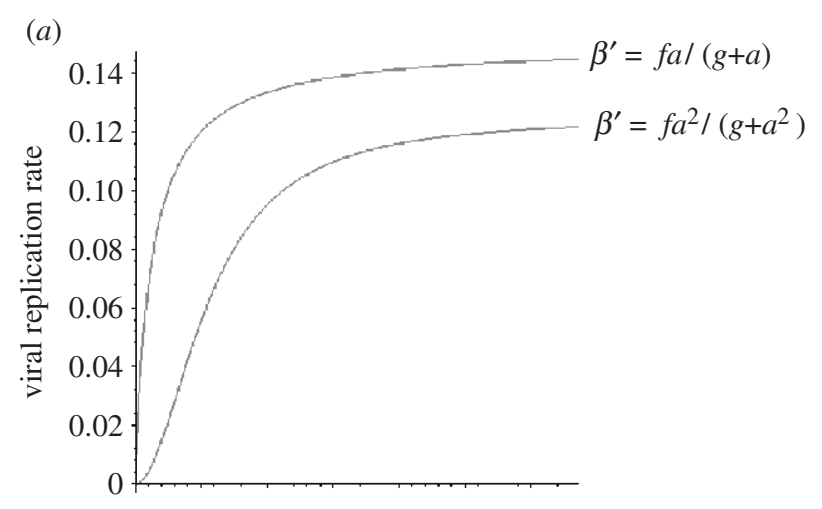

evolve towards such a disease-causing phenotype. In such models, the virus evolves towards the highest basic reproductive ratio, $R_{0}$, a measure of viral spread (Anderson \& May 1991). In the most basic model outlined in $\S 2$, the virus with the highest basic reproductive ratio is non-cytopathic (Nowak \& May 2000), and this phenotype cannot deplete the target cell population. However, this is due to the unrealistic assumption that average viral cytopathicity, $a$, and average replication rate, $\beta$, are independent variables. Hence, the less cytopathic the virus, the longer lived the infected cell and the higher the number of viruses produced by the infected cell during its lifespan. Under the more realistic assumption that these parameters are coupled, the relationship between viral cytopathicity and replication can be given by $\beta^{\prime}=f a /(g+a)$, where an increase in the viral cytopathicity asymptotically correlates with an increased replication rate (figure $1 a$ ). The same general relationship can be described by other mathematical expressions, although the results described here remain robust (see electronic supplementary material, $\S$ b). Such relationships have commonly been used in studies that examine the evolution of parasite virulence (Anderson \& May 1982; May \& Nowak 1994; Ebert \& Herre 1996; Frank 1996; Ebert \& Mangin 1997), and the following is an application of this to in vivo HIV dynamics. Under these assumptions, a virus with an intermediate cytopathicity has the maximal basic reproductive ratio (figure $1 b$ ). We denote this by $a_{\mathrm{fit}}$. At lower viral cytopathicities, the replication rate of the virus is reduced under the assumptions outlined above; at higher viral cytopathicities, the relationship between viral cytopathicity and replication becomes weak, and an increased viral cytopathicity reduces the burst size of infected cells.

For the virus to evolve to a pathogenic phenotype, the level of cytopathicity that causes maximal pathology, $a_{\text {path }}$, should be around the same level as the one which maximizes the basic reproductive ratio and thus replicative fitness, $a_{\text {fit }}$ (figure 2). In the model, these parameters are given by $a_{\text {path }}=(\lambda f)^{1 / 2}-d$ and $a_{\text {fit }}=(g d)^{1 / 2}$. The condition $a_{\text {path }}=a_{\text {fit }}$ is achieved if $g=\left[d^{2}-2 d(\lambda f)^{1 / 2}+\lambda f\right] / d$. However, we further find that under this condition, the maximal basic reproductive ratio of the virus $R_{0 \text { max }}=1$ (figure 2). This means that the virus cannot grow. If $a_{\mathrm{fit}}>$ $a_{\text {path }}$, then $R_{0 \text { max }}<1$ (figure 2). Again, the virus cannot grow under this condition. The virus can grow (i.e. $R_{0 \text { max }}>1$ ) only if $a_{\text {fit }}<a_{\text {path }}$ (figure 2); that is, the virus evolves to a level of cytopathicity that is too low to induce cell depletion. These results have been obtained assuming a specific mathematical dependency between the parameters $\beta^{\prime}$ and $a$. In fact, it can be shown that $R_{0 \text { max }}=1$ if $a_{\text {fit }}=a_{\text {path }}$ for any dependency between $\beta^{\prime}$ and $a$ (see electronic supplementary material, $\S b$ ).

In the earlier phases of HIV infection, less cytopathic and non-pathogenic strains prevail and out-compete more cytopathic strains, which tend to correlate with cell depletion (Schuitemaker 1994; van't Wout et al. 1994). Therefore, we have $a_{\mathrm{fit}}<a_{\text {path }}$, in accordance with the model. This brings up a paradox: for the virus population to attain a disease-causing phenotype, it has to evolve towards a reduced basic reproductive ratio, $R_{0}$, where the level of viral cytopathicity is approximately $a_{\text {path }}$ (figure 2 ). In the basic modelling framework, evolution towards a reduced basic reproductive ratio is impossible since it correlates with reduced replicative fitness. 
(a)

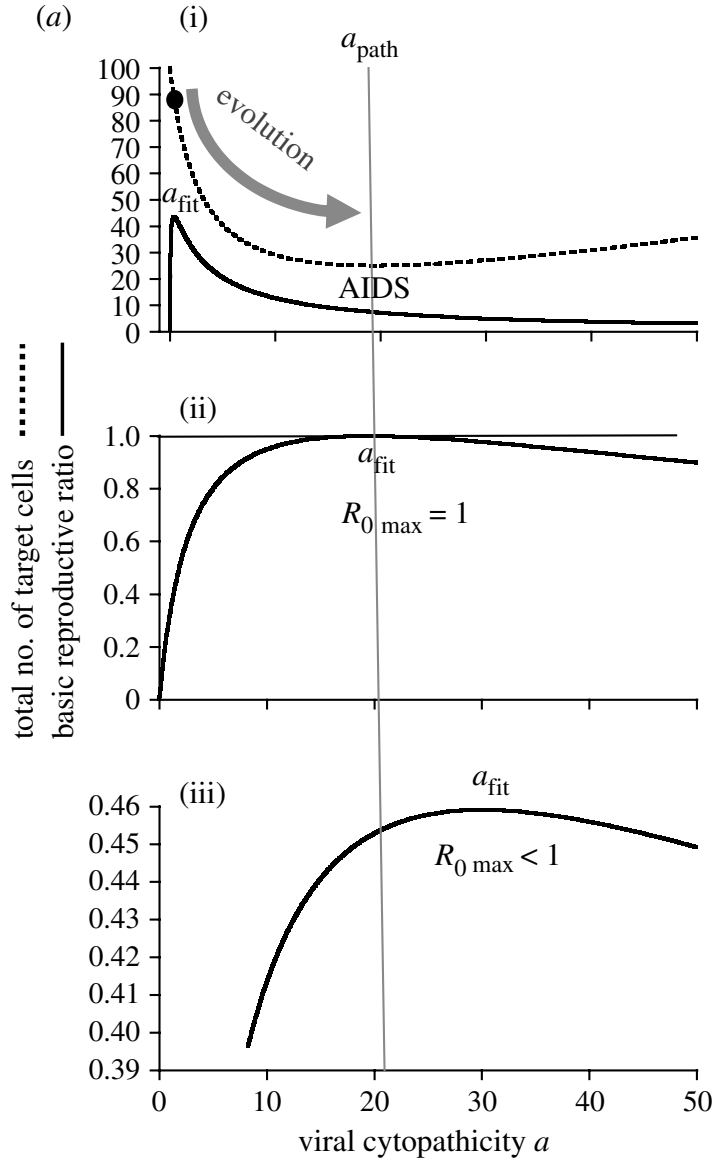

(b)

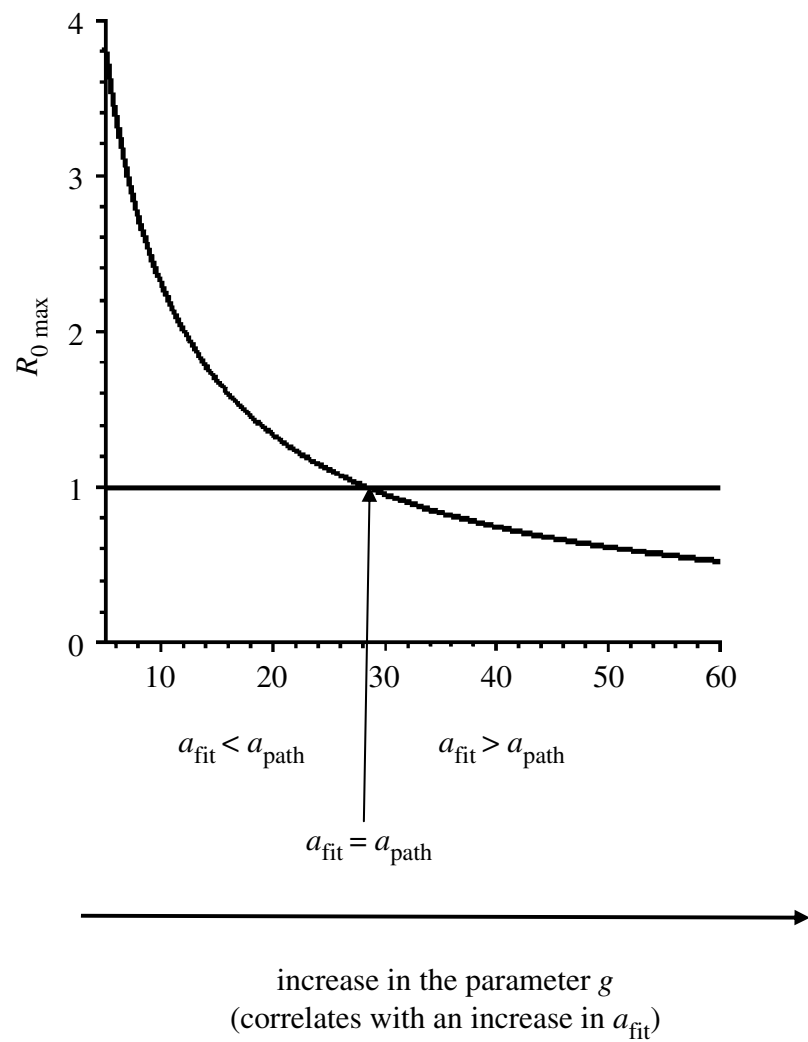

Figure 2. Evolution of viral cytopathicity in the absence of co-infection, using model (2.1). (a) The level of viral cytopathicity that maximizes the basic reproductive ratio of the virus $\left(a_{\text {fit }}\right)$ cannot coincide with the level of cytopathicity that maximizes pathology $\left(a_{\text {path }}\right)$. (ii) If $a_{\mathrm{fit}}=a_{\text {path }}$, then the peak value of $R_{0}, R_{0 \text { max }}=1$, and the virus cannot spread. (iii) If $a_{\mathrm{fit}}>a_{\mathrm{path}}$, then $R_{0 \text { max }}<1$ and the virus again cannot spread. However, (i) if $a_{\mathrm{fit}}<a_{\mathrm{path}}$, then $R_{0}$ is greater than 1 over a wide parameter region, and the virus can spread. Hence, this is the only possibility. Therefore, for HIV to evolve towards maximal pathology, the virus has to evolve from the highest $R_{0}$ to a reduced value of $R_{0}$. (b) The same information is summarized more concisely in this plot, showing the value of the maximal basic reproductive ratio of the virus $R_{0 \text { max }}$ as a function of $a_{\mathrm{fit}}$ (determined by the parameter $g$ in the model, see \$2). The value where $a_{\mathrm{fit}}=a_{\text {path }}$ is indicated by the arrow. If $\mathrm{a}_{\mathrm{fit}}<a_{\text {path }}$, then $R_{0 \max }>1$ and the infection is viable. If $a_{\mathrm{fit}}=a_{\mathrm{path}}$, then $R_{0 \text { max }}=1$ and the infection cannot spread within the host. If $a_{\mathrm{fit}}>a_{\text {path }}$, then $R_{0 \text { max }}<1$ and the infection goes extinct. Parameters were chosen as follows: (a) $\lambda=100, d=3, f=5$, (i) $g=0.05$, (ii) $g=124$, (iii) $g=300$; (b) $\lambda=10$, $d=1, f=4$. Parameters were chosen for illustrative purposes. Units are arbitrary. Dotted line, total number of target cells; solid line, basic reproductive ratio.

\section{(b) Effect of co-infection}

Interestingly, evolution towards a reduced basic reproductive ratio is possible if we include the assumption of co-infection (two or more viruses can infect the same cell) into the model, which has been clearly documented to occur with HIV (Jung et al. 2002; Dang et al. 2004; Levy et al. 2004; Chen et al. 2005). We extend the basic virus infection model to include two strains of viruses. The model is based on previous epidemiological work by Levin \& Pimentel (1981). The model contains the following new variables: $y_{1}$ are cells infected with strain $1 ; y_{2}$ are cells infected with strain 2 ; and $y_{12}$ are cells infected with both viruses. For convenience we assume that $y_{2}$ is more cytopathic than $y_{1}$ (i.e. $a_{2}>a_{1}$ ). Host cells can become infected by strain 1 with a rate $\beta_{1}^{\prime}$ and strain 2 with a rate $\beta_{2}^{\prime}$. Virus strain 1 can be produced by cells $y_{1}$ and $y_{12}$. Virus strain 2 can be produced by cells $y_{2}$ and $y_{12}$. Cells infected only by strain 1 are killed with a rate $a_{1}$ and those infected only by strain 2 are killed with a rate $a_{2}$. For co-infected cells, we assume the occurrence of dominance: the cells die with a rate determined by the more cytopathic strain, $a_{2}$. Thus, the burst size of the less cytopathic strain is reduced. Mathematical details are given in $\$ 2$. Note that we have no experimental insights regarding the relationship between the death rate of an infected cell and the number of viruses with which it is infected. If co-infected cells produce more virus than singly infected cells, it is possible that the death rate of co-infected cells is in fact larger than that induced by the more cytopathic virus. This would also keep with our general notion that there is a relationship between the rates of viral replication and cell death. As described in the electronic supplementary material, we have explored this scenario by numerical simulations and found that it underscores the results derived from the model described here. Note that mathematical models co-infection dynamics have been explored before (Dixit \& Perelson 2005) although the focus of the research was very different.

Assume that the basic reproductive ratio of each virus in isolation is greater than 1, that is, in isolation each virus would be able to sustain an infection. From now on, we assume that the cytopathicity of virus strain 1 is 
Table 1. Summary of the properties of the mathematical models that do and do not take into account the possibility of co-infection, i.e. the infection of a cell by multiple virus particles.

model without co-infection

competitive exclusion only outcome

strain with highest $R_{0}$ wins

fittest strain not the most pathogenic one

approximately $a_{1}=a_{\mathrm{fit}}$. We further assume that virus strain 2 has a higher degree of cytopathicity $\left(a_{2}>a_{\mathrm{fit}}\right)$, which enables it to induce more pronounced target cell depletion. As a result of these assumptions, virus strain 2 is always less fit than virus strain 1 . We ask under which conditions the more cytopathic virus strain 2 can persist in the virus population. The less cytopathic virus strain 1 always persists. The more cytopathic virus strain 2 persists if $\beta_{2}^{\prime}\left(x+y_{1}\right) /\left(a_{2}+d\right)>1$, where $x=\left(a_{1}+d\right) / \beta_{1}^{\prime}$ and $y_{1}=\lambda /$ $\left(a_{1}+d\right)-d / \beta_{1}^{\prime}$. In other words, the less cytopathic strain can exclude the more cytopathic one but not vice versa. If the above condition is fulfilled, a more cytopathic strain can coexist with a less cytopathic strain, even though it has a lower replicative fitness and would be driven extinct in the absence of co-infection. The reason for the coexistence is as follows. Because the co-infected cells die with the rate determined by the more cytopathic virus, the advantage of being less cytopathic is largely abolished in the context of co-infection. Moreover, when co-infection is possible, cells infected with strain 1 remain targets for infection with strain 2 and vice versa, thus increasing the total number of susceptible cells. The persistence of competitively inferior pathogens has also been observed in epidemiological models that describe the spread of a pathogen through a population of hosts and that take into account the infection of a host by multiple pathogens (Levin \& Pimentel 1981; Levin 1983a, $b$; Nowak \& May 1994).

Note that although there is CD4 down-modulation following infection with the first virus, this takes a couple of days, leaving the cell susceptible to reinfection during this window. Levy et al. (2004) showed that during virus replication in $\mathrm{CD} 4+\mathrm{T}$ cells and human thymocytes in the SCID-hu thy/liv mouse model, co-infection occurs without apparent functional inhibition. Thus, the total number of infectible targets for each strain is increased by allowing co-infection.

The result that a more cytopathic strain with reduced replicative fitness can coexist with a fitter virus strain has important implications. Even if the more cytopathic strain is subdominant, its presence can still result in a sharp decline of the overall number of T cells. By how much the T-cell count is expected to fall depends on the level of cytopathicity of the new strain. The effect of co-infection on virus competition and pathology is summarized in table 1 .

\section{(c) Co-infection, virus load and the development} of $\boldsymbol{A I D S}$

Co-infection has been documented to occur with HIV (Jung et al. 2002; Dang et al. 2004; Levy et al. 2004; Chen et al. 2005). If co-infection promotes the emergence of less fit and more cytopathic strains that induce disease, why does transmission to a new host always 'reset the disease clock', i.e. result in the prevalence of less cytopathic HIV strains that do not cause disease, but keep the infection asymptomatic? The answer might be immune responses. model with co-infection

coexistence of virus strains possible

strains with reduced replicative fitness can persist

persistence of less fit strains can cause pathology
For co-infection to be a significant force in the dynamics of HIV evolution and disease progression, virus load needs to be sufficiently high such that cells are frequently infected with more than one virus. If virus load is suppressed by immune responses, co-infection is a rare event and does not drive the dynamics. We incorporate a general mathematical model of immune responses (De Boer \& Perelson 1998; Nowak \& May 2000; Perelson 2002) into our framework. According to this model, immune responses expand upon antigenic stimulation (by both virus strains) and can fight the virus population by both lytic and non-lytic means. Details of the equations and parameters are given in $\S 2$. This model is a phenomenological description of antigen-driven immune expansion and can be used to describe T- and B-cell responses. Our arguments hold true for any model as long as the presence of immune responses can lead to reduced virus load.

If the strength of immunity lies below a threshold, the dynamics are, in principle, the same as in the scenario where no immune responses are present. In other words, co-infection allows the emergence of more cytopathic and less fit HIV strains that could lead to the depletion of the CD4 T-cell population. If the strength of immunity is higher and lies above a threshold, however, the dynamics change fundamentally. Now, virus load is relatively low and co-infection is a rare event. Under this condition, the behaviour of the model approaches the dynamics observed in the absence of co-infection. In other words, more pathogenic and less fit viruses cannot emerge, and the virus is expected to evolve towards its highest basic reproductive ratio, where the viral cytopathicity is too low to induce AIDS ( $a=a_{\mathrm{fit}}<a_{\mathrm{path}}$ ). The virus population is also predicted to be more homogeneous and the patient to remain asymptomatic. This behaviour has been found by extensive numerical simulations. Owing to the complexity of the model, an analytic expression for the immune strength threshold could not be attained.

\section{(d) Overall picture of disease progression}

These results translate into the following overall picture of HIV disease progression, summarized with computer simulations in figure 3 and schematically in figure 4 . During the acute phase where virus loads are high, the cytopathic strain persists and induces a temporary drop in the CD4 T-cell count, which has been observed to occur especially in the gut-associated lymphoid tissue (Veazey et al. 1998). However, in the post-acute phase, relatively strong immunity is initially present, and this could account for the selection of less cytopathic strains that are characterized by $a=a_{\text {fit }}$. Less fit and more cytopathic virus strains go extinct, and the patient remains asymptomatic. During progression, anti-viral immunity gradually loses its ability to control the virus owing to a variety of reasons, such as the killing of HIV-specific helper cells (Rosenberg et al. 1997; Kalams et al. 1999) and viral 
(a)
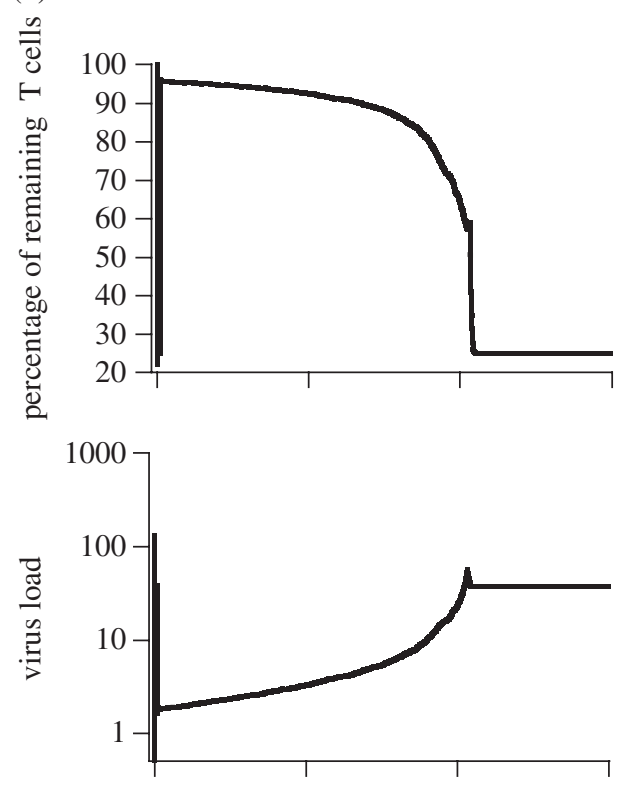

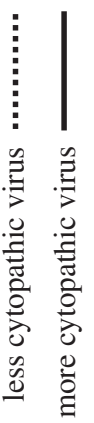

(b)
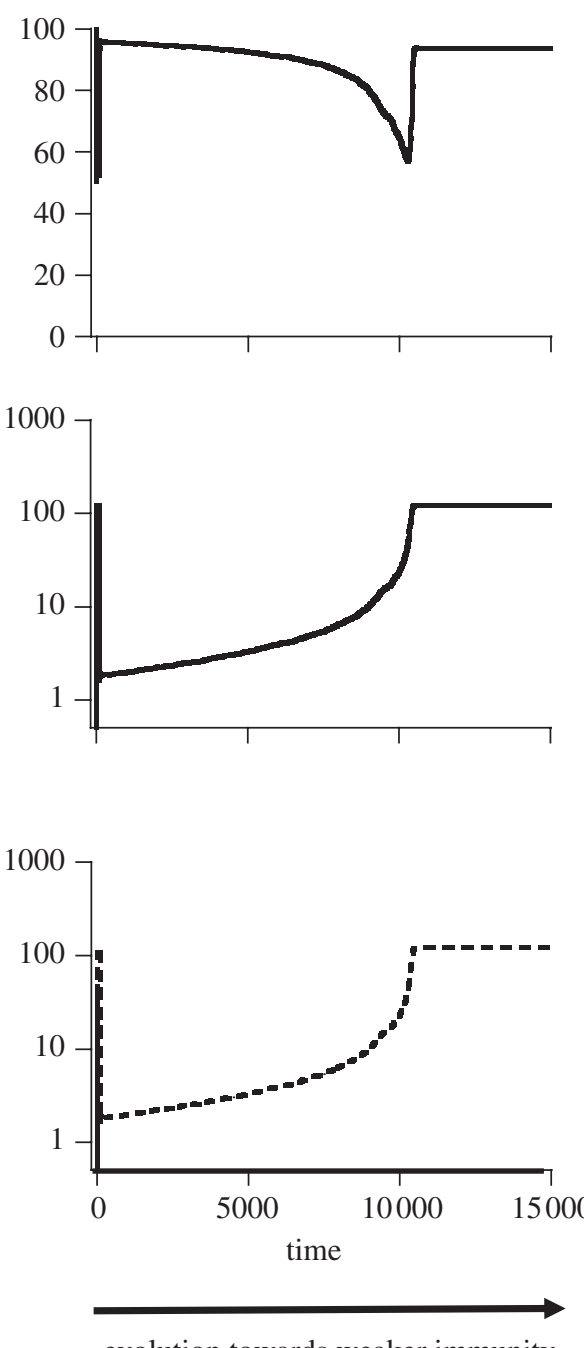

evolution towards weaker immunity over time

Figure 3. Simulation of disease progression (a) assuming that co-infection can occur at sufficient levels and $(b)$ assuming that co-infection is blocked, using model (2.2). These graphs plot the percentage of CD4 cells and virus load over time, assuming that the immune responsiveness, $c$, is reduced over time in a stochastic fashion. We start with the presence of a less cytopathic virus and assume that, at regular time intervals, there is a chance that a more cytopathic virus with a reduced basic reproductive ratio is created by mutation. (a) Co-infection dynamics: during the acute phase the virus replicates to high levels. Both virus strains can be present during the acute phase. The more cytopathic strain contributes to a temporary but significant drop in the CD4 cell count during this phase. As immune responses rise, virus load drops and the more cytopathic strain is out-competed by the less cytopathic strain with higher replicative fitness. This is because at low virus loads the chance of co-infection occurring is relatively low. Consequently, the CD4 cell count returns to relatively high levels. Over time, the immune response weakens and virus load rises. Once virus load has risen beyond a critical threshold, a significant number of cells will be co-infected. This allows the more cytopathic strain with a reduced basic reproductive ratio to invade. This induces a relatively sharp and permanent drop in the CD4 cell count and could correspond to the development of AIDS. (b) Same simulation assuming that co-infection is blocked. The initial dynamics are identical. However, as immunity weakens and virus load rises to high levels, there is a fundamental difference: the more cytopathic strain with a reduced basic reproductive ratio cannot invade, and the CD4 T-cell count is maintained at relatively high levels in the long term, despite the high viral loads. We observe a temporary drop in the CD4 cell count as the immune system weakens and virus load rises. This is because limited immune-mediated lysis in the presence of relatively high virus load can result in the killing of many CD4 cells, a process called CTL-induced pathology (Wodarz et al. 2002). This effect, however, is not expected to be strong if non-lytic immunity also fights the infection (Wodarz et al. 2002). Parameters were chosen as follows: $\lambda=100, d=3, f=5, g=0.05, a_{1}=0.2, a_{2}=20, b=0.05, p=1$, $\varepsilon=1$. Initial value of $c=1$; at regular time intervals, the value of $c$ is reduced by an amount $\Delta=0.001$ with a probability of $q=0.01$. Parameters were chosen for illustrative purposes. Units are arbitrary. Dotted line, less cytopathic virus; solid line, more cytopathic virus.

escape from immune responses (Nowak et al. 1991; Phillips et al. 1991; Goulder et al. 1997; McMichael \& Phillips 1997; Price et al. 1997; Kimata et al. 1999). Once the strength of immunity has fallen below a threshold level, virus load becomes sufficiently high such that co-infection is a frequent event and a driving force of the dynamics. In fact, it has been demonstrated experimentally that the amount of co-infection goes up according to the square of virus load (Levy et al. 2004). Now, more cytopathic and less fit virus strains can emerge and this can 
(a)

low virus load

cytopathic viruses with redcuced replicative fitness cannot emerge

asymptomatic

(b) acute

phase

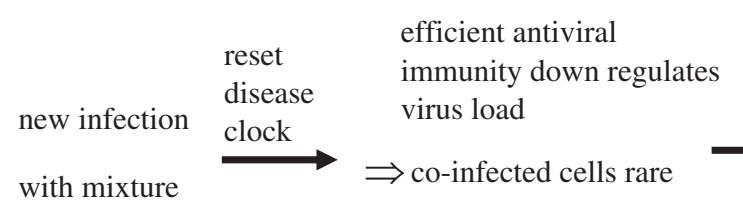
cytopathic strains with reduced

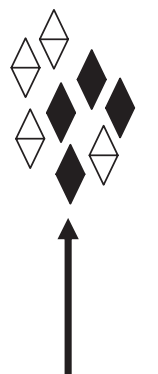

selection against replicative fitness

asymptomaticearly phase

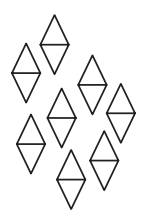

transmission

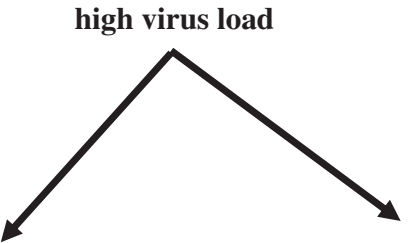

co-infection occurring at significant levels

co-infection occurring at reduced levels

cytopathic viruses with reduced replicative fitness emerge

cytopathic viruses with reduced replicative fitness cannot emerge

\section{AIDSS asymptomatic}

late phase/

AIDS related symptoms

weakened immunity leads to higher virus load

$\Rightarrow$ co-infection frequent

cytopathic strains with reduced replicative fitness emerge and cause disease

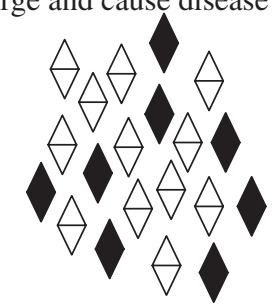

Figure 4. Schematic that summarizes the basic insights gained from the mathematical model. (a) At low virus loads, the more cytopathic strain with a reduced basic reproductive ratio cannot emerge because even if co-infection can take place it is a rare event. This keeps the infection asymptomatic. At higher virus loads we have two possible outcomes. If co-infection can take place at sufficient levels, a more cytopathic strain with a reduced basic reproductive ratio can emerge, and this can result in depletion of the CD4 cell count and the development of AIDS. On the other hand, if co-infection occurs at reduced levels, more cytopathic strains with a reduced basic reproductive ratio cannot emerge, and so the infection remains asymptomatic despite high virus loads. This could occur in naturally SIV-infected monkeys, and could be a goal for therapy. (b) Schematic of the implications for HIV disease progression. A weakening of the immune responses can increase the frequency of co-infection and allow the rise of more cytopathic virus strains that have a reduced basic reproductive ratio and can induce AIDS. If a mix of virus strains is transmitted to a new host, the disease clock is reset, i.e. more cytopathic strains go extinct and the less cytopathic ones prevail, keeping the infection asymptomatic. This is because immune responses are once again strong in the new host, which results in a drop of virus load, infrequent co-infection, and thus selection against more cytopathic and disease-inducing strains that have a lower basic reproductive ratio.

lead to the depletion of the CD4 T-cell population and the development of AIDS (figures 3 and 4). If co-infection occurred at reduced levels, such cytopathic virus strains would not emerge, and this would result in the absence of disease, even at high virus loads (figure 3 ). In this case, virus load could still increase over time during the infection, for example, as a result of immune escape, but the high virus load would not induce AIDS (figure 3).

If the virus gets transmitted from one host to another, immunity will once again be strong in the new host in the post-acute phase (figure 4). Consequently, co-infection is again a rare event. This once more results in the selection of virus strains, which are not cytopathic enough to induce
AIDS $\left(a=a_{\mathrm{fit}}\right)$, and in the extinction of strains that are associated with the onset of disease. Hence, the clock of progression is reset and the disease is initially asymptomatic (figure 4).

Note that in this model, the escape from immune responses is not sufficient to induce AIDS. Escape from immune responses results in high virus loads. High virus loads, in turn, may or may not result in the development of AIDS. This depends on the level of co-infection that occurs, which determines whether disease-inducing virus strains can emerge or not.

In the context of immune responses, it is important to clarify the statement that AIDS develops because the 
virus evolves towards reduced replicative fitness. For co-infection to be a significant driving force of the dynamics, first the virus needs to escape immune responses or weaken immune responses against itself. Obviously, this increases overall fitness of the viral population. However, once the immune response is weakened beyond a critical point and co-infection reaches a threshold level, then virus strains with reduced replicative fitness can emerge and induce an AIDS-defining level of CD4 depletion. By reduced replicative fitness, we mean a reduction in the basic reproductive ratio of the virus, $R_{0}$, defined in the absence of co-infection, but sustainable only by the presence of co-infection made possible by weakened immunity. These concepts are explained in figure 4 .

\section{(e) Lack of disease in naturally SIV-infected monkeys}

These results might also shed light on the reasons for the lack of AIDS in monkeys that are naturally infected with SIV, and might open up new strategies for therapy. Naturally infected monkeys can show high viral loads, virus diversity and lack of strong immune responses to SIV (Goldstein et al. 2000; Broussard et al. 2001; Silvestri et al. 2003)_factors associated with AIDS in HIV-infected patients. While the reasons for this remain unclear and subject to speculation, it is possible that in natural SIV infection, co-infection occurs at reduced levels. It is possible that either the virus or the host or both have evolved mechanisms that reduce the amount of co-infection. In this case, the virus would, according to our model, evolve to a phenotype that is not cytopathic enough to induce AIDS. HIV already has the ability to block co-infection, but only after the cell has been infected for a day or two. It is possible that this time window is significantly shorter in natural SIV infection. More experimental research is needed to investigate the molecular mechanisms that allow and prevent co-infection, and to compare natural SIV infection with pathogenic SIV/HIV infection in this respect. This also has implications for treatment strategies. If co-infection could be inhibited by drugs, a progressing patient could be transformed into a disease-free virus carrier, a state similar to that observed in naturally infected monkeys.

\section{(f) Evolution of replicative fitness in SIVIHIV}

The role of co-infection in HIV disease progression has received a lot of attention recently. The main line of thinking is that co-infection promotes the generation of viral diversity and in vivo evolution, because it leads to the occurrence of recombination inside infected cells (Jung et al. 2002; Dang et al. 2004; Levy et al. 2004; Chen et al. 2005). This was, however, not the focus of our investigation. Instead, we described an additional effect of co-infection that might be crucial for understanding HIV disease progression: it allows cytopathic virus strains with reduced replicative fitness to emerge in late stages of the disease. Without this, AIDS might not be able to develop. Interestingly, a recent theoretical study argued that the ability of HIV to undergo recombination could lead to a reduction in overall viral fitness under specific conditions (Bretscher et al. 2004).

Our conclusion about viral evolution towards reduced replicative fitness needs to be tested. While the model suggests that the emergence of more cytopathic/less fit virus strains might be needed for T-cell depletion, it still leaves open the logical possibility that AIDS can occur without this requirement. Our argument is based on the assumption that for AIDS to develop, the degree of viral cytopathicity needs to be approximately $a=a_{\text {path }}$ because this correlates with maximal target cell depletion. However, even less extensive target cell depletion at $a=a_{\text {fit }}$ might, in principle, be sufficient to induce symptoms of AIDS, especially if the virus replicates with a fast rate. Thus, we need to investigate whether in late stages of the disease, virus variants exist which are less fit and have a lower basic reproductive ratio in the absence of immune responses when compared with virus variants isolated from earlier stages of the disease. This can be assessed to some degree from previously published experimental data.

SIV has been shown to evolve from a relatively slowly replicating and mildly cytopathic strain during early infection (called SIVMneCL8) to a faster replicating and cytopathic strain (called SIVMne170) at later stages of the infection (Kimata \& Overbaugh 1997). Using CEM $\times 174$ cells, recent in vitro competition experiments demonstrated that the early and mildly cytopathic strain SIVMneCL8 was more fit than the late and cytopathic strain SIVMne170 (Voronin et al. 2005). The heightened cytopathicity of the late strain conferred a fitness disadvantage to the virus, and the replication rate was not sufficiently faster to compensate for this disadvantage. This study showed for the first time that virus strains recovered from late infection could be less fit than virus strains recovered from early infection, at least as defined by the parameters and experimental conditions of this in vitro experimental system. It supports our notion that the induction of AIDS might entail evolution towards reduced viral replicative fitness. The argument has been made, however, that this experimental result was due to the long natural lifespan of the cells in culture (Voronin et al. 2005). It was argued that in an immune-mediated high-turnover environment, infected cells are killed by CTL before the virus gets a chance to kill them, rendering viral cytopathicity an insignificant determinant of viral fitness (Voronin et al. 2005). This was supported by a further set of experiments in which the lifespan of the cells in culture was cut short artificially by removing cells. In this case, the late and cytopathic strain SIVMne170 proved to be fitter. However, a mathematical formulation and parameter estimates (Wodarz 2007) suggest that a CTL-mediated high-turnover environment in vivo is unlikely to select for the more cytopathic strain during later stages of the disease, especially given that CTL-mediated immunity becomes less effective during disease progression. Apart from this, the relative rates of virus-induced and immune-mediated death of infected cells are not known, and it is unclear whether CTL kills infected cells before the virus has a chance to do so or not. In summary, the experimental observation is that without further manipulation of the culture, the late diseaseinducing strain was less fit than the early benign strain, and this is an interesting finding in the context of the theory presented here.

While we have mainly discussed the in vivo evolution of SIV, there are also some data that investigate the replicative fitness of HIV (Quinones-Mateu et al. 2000; Troyer et al. 2005), although their interpretation is less clear. HIV derived from patients with accelerated 
progression to AIDS was found to be fitter in vitro than virus isolated from long-term survivors. In general, in vitro viral fitness seemed to correlate with virus load and viral diversity. However, the interpretation of these results is complicated. First, comparing viruses isolated from longterm survivors with those isolated from patients with accelerated progression might not reflect the evolution of a virus population within one host over time. For instance, the virus in the long-term survivor might be characterized by some defect that renders it more benign and thus generally less fit. Another difficulty is that the in vitro competition experiments involved the regular addition of fresh susceptible cells to the culture. If the number of target cells never becomes limiting, the measurements of relative fitness can be altered in an unrealistic way. The theory presented here, however, should encourage more detailed investigations regarding the in vivo fitness of SIV and HIV at different stages of the disease process.

This work was funded in part by NIH grant 1R01AI058153$01 \mathrm{~A} 2$.

\section{REFERENCES}

Anderson, R. M. \& May, R. M. 1982 Coevolution of hosts and parasites. Parasitology 85(Pt 2), 411-426.

Anderson, R. M. \& May, R. M. 1991 Infectious diseases of humans. Oxford, UK: Oxford University Press.

Antia, R., Ganusov, V. V. \& Ahmed, R. 2005 The role of models in understanding CD8+ T-cell memory. Nat. Rev. Immunol. 5, 101-111. (doi:10.1038/nri1550)

Bretscher, M. T., Althaus, C. L., Muller, V. \& Bonhoeffer, S. 2004 Recombination in HIV and the evolution of drug resistance: for better or for worse? Bioessays 26, 180-188. (doi:10.1002/bies.10386)

Broussard, S. R., Staprans, S. I., White, R., Whitehead, E. M., Feinberg, M. B. \& Allan, J. S. 2001 Simian immunodeficiency virus replicates to high levels in naturally infected African green monkeys without inducing immunologic or neurologic disease. F. Virol. 75, 2262-2275. (doi:10.1128/JVI.75.5.2262-2275.2001)

Chen, J., Dang, Q., Unutmaz, D., Pathak, V. K., Maldarelli, F., Powell, D. \& Hu, W. S. 2005 Mechanisms of nonrandom human immunodeficiency virus type 1 infection and double infection: preference in virus entry is important but is not the sole factor. F. Virol. 79, 4140-4149. (doi:10.1128/JVI.79.7.4140-4149.2005)

Dang, Q., Chen, J., Unutmaz, D., Coffin, J. M., Pathak, V. K., Powell, D., KewalRamani, V. N., Maldarelli, F. \& $\mathrm{Hu}$, W. S. 2004 Nonrandom HIV-1 infection and double infection via direct and cell-mediated pathways. Proc. Natl Acad. Sci. USA 101, 632-637. (doi:10.1073/pnas. 0307636100)

De Boer, R. J. \& Perelson, A. S. 1998 Target cell limited and immune control models of HIV infection: a comparison. f. Theor. Biol. 190, 201-214. (doi:10.1006/jtbi.1997.0548)

Dixit, N. M. \& Perelson, A. S. 2005 HIV dynamics with multiple infections of target cells. Proc. Natl Acad. Sci. USA 102, 8198-8203. (doi:10.1073/pnas.0407498102)

Ebert, D. \& Herre, E. A. 1996 The evolution of parasitic diseases. Parasitol. Today 12, 96-101. (doi:10.1016/01694758(96)80668-5)

Ebert, D. \& Mangin, K. L. 1997 The influence of host demography on the evolution of virulence of a microsporidian gut parasite. Evolution 51, 1828-1837. (doi:10. 2307/2411005)

Frank, S. A. 1996 Models of parasite virulence. Q. Rev. Biol. 71, 37-78. (doi:10.1086/419267)
Goldstein, S., Ourmanov, I., Brown, C. R., Beer, B. E., Elkins, W. R., Plishka, R., Buckler-White, A. \& Hirsch, V. M. 2000 Wide range of viral load in healthy African green monkeys naturally infected with simian immunodeficiency virus. F. Virol. 74, 11 744-11 753. (doi:10. 1128/JVI.74.24.11744-11753.2000)

Goulder, P. J. et al. 1997 Late escape from an immunodominant cytotoxic T-lymphocyte response associated with progression to AIDS. Nat. Med. 3, 212-217. (doi:10. 1038/nm0297-212)

Hirsch, V. M. 1999 Evolution of the fittest ends in tragedy. Nat. Med. 5, 488-489. (doi:10.1038/8369)

Jung, A., Maier, R., Vartanian, J. P., Bocharov, G., Jung, V., Fischer, U., Meese, E., Wain-Hobson, S. \& Meyerhans, A. 2002 Multiply infected spleen cells in HIV patients. Nature 418, 144. (doi:10.1038/418144a)

Kalams, S. A., Buchbinder, S. P., Rosenberg, E. S., Billingsley, J. M., Colbert, D. S., Jones, N. G., Shea, A. K., Trocha, A. K. \& Walker, B. D. 1999 Association between virus-specific cytotoxic T-lymphocyte and helper responses in human immunodeficiency virus type 1 infection. F. Virol. 73, 6715-6720.

Kimata, J. T. \& Overbaugh, J. 1997 The cytopathicity of a simian immunodeficiency virus Mne variant is determined by mutations in Gag and Env. F. Virol. 71, 7629-7639.

Kimata, J. T., Kuller, L., Anderson, D. B., Dailey, P. \& Overbaugh, J. 1999 Emerging cytopathic and antigenic simian immunodeficiency virus variants influence AIDS progression. Nat. Med. 5, 535-541. (doi:10. 1038/8414)

Levin, S. A. $1983 a$ Coevolution. In Population biology (eds H. I. Freedman \& C. Strobeck), pp. 328-334. Berlin, Germany: Springer.

Levin, S. A. $1983 b$ Some approaches to the modeling of coevolutionary interactions. In Coevolution (ed. M. Nitecki), pp. 21-65. Chicago, IL: University of Chicago Press.

Levin, S. A. \& Pimentel, D. 1981 Selection of intermediate rates of increase in parasite-host systems. Am. Nat. 117, 308-315. (doi:10.1086/283708)

Levy, D. N., Aldrovandi, G. M., Kutsch, O. \& Shaw, G. M. 2004 Dynamics of HIV-1 recombination in its natural target cells. Proc. Natl Acad. Sci. USA 101, 4204-4209. (doi:10.1073/pnas.0306764101)

May, R. M. \& Nowak, M. A. 1994 Superinfection, metapopulation dynamics, and the evolution of diversity. F. Theor. Biol. 170, 95-114. (doi:10.1006/jtbi.1994.1171)

McMichael, A. J. \& Phillips, R. E. 1997 Escape of human immunodeficiency virus from immune control. Annu. Rev. Immunol. 15, 271-296. (doi:10.1146/annurev.immunol. 15.1.271)

Nowak, M. A. \& May, R. M. 1994 Superinfection and the evolution of parasite virulence. Proc. R. Soc. B 255, 81-89. (doi:10.1098/rspb.1994.0012)

Nowak, M. A. \& May, R. M. 2000 Virus dynamics. Mathematical principles of immunology and virology. Oxford, UK: Oxford University Press.

Nowak, M. A., Anderson, R. M., McLean, A. R., Wolfs, T. F. W., Goudsmit, J. \& May, R. M. 1991 Antigenic diversity thresholds and the development of AIDS. Science 254, 963-969. (doi:10.1126/science.1683006)

Perelson, A. S. 2002 Modelling viral and immune system dynamics. Nat. Rev. Immunol. 2, 28-36. (doi:10.1038/ nri700)

Phillips, R. E. et al. 1991 Human immunodeficiency virus genetic variation that can escape cytotoxic $\mathrm{T}$ cell recognition. Nature 354, 453-459. (doi:10.1038/ 354453a0)

Price, D. A., Goulder, P. J., Klenerman, P., Sewell, A. K., Easterbrook, P. J., Troop, M., Bangham, C. R. \& Phillips, 
R. E. 1997 Positive selection of HIV-1 cytotoxic T lymphocyte escape variants during primary infection. Proc. Natl Acad. Sci. USA 94, 1890-1895. (doi:10.1073/ pnas.94.5.1890)

Quinones-Mateu, M. E., Ball, S. C., Marozsan, A. J., Torre, V. S., Albright, J. L., Vanham, G., van Der Groen, G., Colebunders, R. L. \& Arts, E. J. 2000 A dual infection/competition assay shows a correlation between ex vivo human immunodeficiency virus type 1 fitness and disease progression. F. Virol. 74, 9222-9233. (doi:10. 1128/JVI.74.19.9222-9233.2000)

Rosenberg, E. S., Billingsley, J. M., Caliendo, A. M., Boswell, S. L., Sax, P. E., Kalams, S. A. \& Walker, B. D. 1997 Vigorous HIV-1-specific CD4+ T cell responses associated with control of viremia. Science 278, 1447-1450. (doi:10.1126/science.278.5342.1447)

Schuitemaker, H. 1994 Macrophage-tropic HIV-1 variants: initiators of infection and AIDS pathogenesis? $\mathcal{F}$. Leukocyte Biol. 56, 218-224.

Silvestri, G., Sodora, D. L., Koup, R. A., Paiardini, M., O’Neil, S. P., McClure, H. M., Staprans, S. I. \& Feinberg, M. B. 2003 Nonpathogenic SIV infection of sooty mangabeys is characterized by limited bystander immunopathology despite chronic high-level viremia. Immunity 18，441-452. (doi:10.1016/S1074-7613(03) 00060-8)
Troyer, R. M. et al. 2005 Changes in human immunodeficiency virus type 1 fitness and genetic diversity during disease progression. F. Virol. 79, 9006-9018. (doi:10. 1128/JVI.79.14.9006-9018.2005)

van't Wout, A. B. et al. 1994 Macrophage-tropic variants initiate human immunodeficiency virus type 1 infection after sexual, parenteral, and vertical transmission. F. Clin. Invest. 94, 2060-2067.

Veazey, R. S. et al. 1998 Gastrointestinal tract as a major site of CD4 + T cell depletion and viral replication in SIV infection. Science 280, 427-431. (doi:10.1126/science.280.5362.427)

Voronin, Y., Overbaugh, J. \& Emerman, M. 2005 Simian immunodeficiency virus variants that differ in pathogenicity differ in fitness under rapid cell turnover conditions. F. Virol. 79, 15 091-15 098. (doi:10.1128/ JVI.79.24.15091-15098.2005)

Wodarz, D. 2007 On the relative fitness of early and late stage Simian immunodeficiency virus isolates. Theor. Popul. Biol. Epub ahead of print. (doi:10.1016/j.tpb.2007.03.005).

Wodarz, D. \& Krakauer, D. C. 2000 Defining CTL-induced pathology: implications for HIV. Virology 274, 94-104. (doi:10.1006/viro.2000.0399)

Wodarz, D., Christensen, J. P. \& Thomsen, A. R. 2002 The importance of lytic and nonlytic immune responses in viral infections. Trends Immunol. 23, 194-200. (doi:10.1016/ S1471-4906(02)02189-0) 


\section{NOTICE OF CORRECTION}

The equations in $\S \S 2 a, b$ and $3 b$ are now presented in the correct form.

1 February 2008 